\title{
\begin{tabular}{l|l} 
pcori). & PATIENT-CENTERED OUTCOMES RESEARCH INSTITUTE \\
RESEARCH SUMMARY
\end{tabular}
}

\section{Comparing Two Methods of Caring for Black and Hispanic Adults with Heart Failure after They Leave the Hospital}

Principal investigator

Renee Pekmezaris, PhD
Organization

Feinstein Institute for Medical Research

\section{What was the research about?}

Heart failure occurs when the heart is unable to pump enough blood to the rest of the body. It can often cause admission to the hospital, especially for people 65 or older. Heart failure can lead to frequent hospital visits, poor quality of life, and death.

In this study, the research team wanted to improve care for black and Hispanic adults with heart failure after they leave the hospital. The team compared two groups for three months.

\section{- Telehealth self-monitoring with a computer} system. The team asked patients to use a device to submit their vital signs, including blood pressure and heart rate, every day. A research nurse reviewed this information within three days. Patients also had weekly video calls with the nurse. During these calls, the nurse discussed the vital signs, asked about symptoms, and listened to the patient's heart and lungs with a digital stethoscope.

- Clinic outpatient management. Patients received usual care from the hospital or their main heart doctor.

The team looked at how many times patients in each group visited the emergency room and had hospital stays. The team also compared quality of life, anxiety, and depression in the two groups.

\section{What were the results?}

During the study, the percentage of patients who went back to the hospital at least once was about the same for the two groups. The average number of times each patient stayed in the hospital for any reason was higher among patients using self-monitoring. However, the number of times patients stayed in the hospital for heart failure or heart disease was about the same for the two groups.

The groups didn't differ in emergency room visits, quality of life, or depression. Patients who received clinic outpatient management showed greater reduction in anxiety than patients in the telehealth self-monitoring group.

\section{Who was in the study?}

The study included 104 adults with heart failure who received care at a hospital in New York that serves people with low incomes. Of these adults, 69 percent were black, and 31 percent were Hispanic. The average age was 60 , and 59 percent were men.

\section{What did the research team do?}

The research team assigned each patient by chance to one of the two groups. Care for patients in both groups began within one week of patients leaving the hospital and continued for three months. For the next two months, patients using telehealth self-monitoring had a video call with a nurse, while the clinic group received care as usual. 
To ask about emergency room visits and hospital stays, a nurse called all patients weekly. The team also looked at medical records for information about these visits and stays. All patients completed a survey about their quality of life at the start and end of the study.

The team worked with a community advisory group during the study. This group included black and Hispanic patients with heart failure, caregivers, patient advocates, and healthcare providers.

\section{What were the limits of the study?}

Half of the patients in the telehealth self-monitoring group didn't submit their vital signs regularly. The selfmonitoring program may not have worked well for them for this reason. The study was small and included patients who received care at only one specialized heart failure clinic. Results may differ for people receiving care at other clinics or hospitals.

Future studies could look at ways to encourage patients to submit their vital signs daily.

\section{How can people use the results?}

Heart failure clinics could use these study results as they are thinking about how to provide care for black and Hispanic patients with heart failure.

To learn more about this project, visit www.pcori.org/Pekmezaris136. 